\title{
Warum definiert Bergson Religion von der Mystik her?
}

\author{
Gerrit Steunebrink \\ Titus Brandsma Insitute \\ Erasmusplein 1, 6525 HT Nijmegen, The Netherlands \\ g.steunebrink@ftr.ru.nl
}

\begin{abstract}
Why Does Bergson Understand Religion From the Perspective of Mysticism?

In his book Bergson makes first of all the distinction between closed societies, with a static religion, and open societies with the dynamic religion. In fact those dynamic religions are the world religions, and they are understood from the perspective of mysticism. Bergson does not accept the opposition between a mystical and a technical worldview. To the contrary, they are related to each other, especially in Christianity, by the virtue of charity. Charity, and therefore technics, is the practical dimension of mysticism. From the perspective of the mystical experience, an intuition of unity with the totality, Bergson tries to rethink the proof of the existence of God, the immortality of man and the theodicy. Modern man, as a technical man represents a special phase in the history of the evolution of nature and its divine 'elan vital'. It is a phenomenon in which nature shows a new dimension of itself. Because the technical dimension enlarges man's capacity of transcendence of the sense of given reality, it creates new possibilities for mystical experience. Modern man needs this mysticism because only mysticism can be the soul within the body of the technical world created by man.
\end{abstract}

\section{Keywords}

mysticism - evolution - nature - world religions - Christianity - modernity technics 
Im Zentrum meiner Auseinandersetzung mit Henri Bergson steht sein spätes Werk Les deux sources de la morale et de la religion (Deutsch:Die beiden Quellen der Moral und der Religion), das 1932 erschien. ${ }^{1}$ Obwohl es lange Zeit in Vergessenheit geraten war, gibt es inzwischen Anzeichen für ein neues Interesse. ${ }^{2}$ Bergsons Reflexionen über Moral und Religion haben unterschwellig deutliche Spuren im Denken des 20. Jahrhunderts hinterlassen. So stammt Poppers berühmte Unterscheidung zwischen einer offenen und einer geschlossenen Gesellschaft von Bergson. Auch Jaspers' Idee einer Achsenzeit in der Entwicklung der Religionen und Philosophien der Menschheit geht zurück auf Bergsons Idee des geschichtlichen Übergangs von einer geschlossenen zu einer offenen Gesellschaft. ${ }^{3}$ Bergson schrieb das Buch unter dem Einfluss des Ersten Weltkrieges und der Gründung des Völkerbundes; Jaspers, den Bergson - so Voegelin - niemals zitiert, ${ }^{4}$ griff die Thematik von Bergson in der Zeit des Zweiten Weltkrieges und der Gründung der Vereinten Nationen wieder auf. Bergson inspirierte aber auch den Kulturanthropologen Lévi-Strauss in seiner Deutung des Totemismusproblems. ${ }^{5}$ Bergsons spätes Werk über Moral und Religion ist ein großes, inspirierendes Buch, das verdient, wieder gelesen zu werden, meines Erachtens vor allem wegen seiner Deutung der Beziehung zwischen der Mystik und dem modernen technischen Zeitalter, ein Thema, das in einer anderen Weise auch Karl Jaspers ausgearbeitet hat.

\section{Wer war Bergson?}

Henri Bergson (1895-1941) war in seiner Zeit ein weltberühmter Philosoph. Seine Vorlesungen waren ein gesellschaftliches Ereignis. Das Auditorium Maximum war immer überfüllt, nicht nur mit Fachphilosophen wie Étienne Gilson, Jean Wahl, Jacques Maritain, sondern auch mit Künstlern wie T.S. Eliott und Charles Péguy. Auch der Schriftsteller und Freund Marcel Proust war von Bergson, der über seine Frau mit ihm verwandt war, beeindruckt. Bis zum Ersten Weltkrieg hatte er seine größten Erfolge. Bergson erhielt 1927 den Nobelpreis für Literatur und starb 1941. Die Berühmtheit Bergsons hat

1 Bergson 1932; dt. 1933. Im Folgenden zitiere ich aus den gesammelten Werken 1970. Im Hinblick auf die zahlreichen Editionen, in denen Bergsons Schrift inzwischen erschienen ist, wird diese nach Kapitel und Paragraphen zitiert. Da der Beitrag durchgängig auf diese Schrift Bergsons Bezug nimmt, erfolgen die Belege zum Teil im Fließtext.

2 Lefebvre 2012.

3 Jaspers 1983 .

4 Gontier 2012; Germino 1974.

5 Lévi-Strauss 2008, pp. $536 \mathrm{ff}$. 
mit seinem Versuch zu tun, in einem vom Positivismus dominierten Zeitalter metaphysische Fragen wieder hoffähig zu machen. Aus diesem Grund erfreute sich Bergson auch einer großen Beliebtheit bei katholischen Dichtern und Denkern, die damals das sogenannte "Renouveau Catholique“, die Bewegung der „Katholischen Erneuerung“ bildeten, zu der Schriftsteller wie Charles Péguy, Léon Bloy und Paul Claudel, aber auch Philosophen wie Jacques Maritain und Etienne Gilson gehörten.

Die enorme Bedeutung von Bergson für die katholische Erneuerung zeigt sich auch an der Lebensgeschichte von Jacques Maritain, der in einer existentiellen Krise, in der er mit seiner Frau Selbstmord begehen wollte, in Bergsons Philosophie einen Ausweg aus der Erfahrung der Sinnlosigkeit fand. Maritain wurde Bergsons Schüler. In der Folge hat Maritain, wie in diesem Kreis üblich, seinen Weg zum Katholizismus (zurück-)gefunden und sich schließlich gegen Bergson gewendet. Bergsons Philosophie wurde 1914 vom Papst verurteilt, man sagt auf Treiben Maritains, der sich Sorgen über Bergsons Beliebtheit in katholischen Kreisen machte. Zur großen Überraschung, auch für Maritain, wollte sich Bergson jedoch in den dreißiger Jahren zum Katholizismus bekehren. Bergson war jüdischer Herkunft, hatte jedoch bereits früh seinen Glauben verloren. Bekehrungen auch aus jüdischen Kreisen gehörten im Übrigen zum Milieu der katholischen Erneuerung, und zwar sowohl in Frankreich als auch in Deutschland. Bergson ließ sich jedoch nicht öffentlich taufen, denn er fühlte den wachsenden Antisemitismus in Europa und wollte sein Volk nicht im Stich lassen. Als die Nazis Frankreich besetzten, stand Bergson Schlange, um sich als Jude registrieren zu lassen. Man holte ihn aus der Reihe. Ein Jahr später starb Bergson an einer Lungenentzündung, eine Folge einer Grippe, die er bei der Registrierung bekommen hatte. Bergson wurde nach seinem Wunsch katholisch beerdigt. ${ }^{6}$

In seinem Werk Les deux sources de la religion et la morale zeigen sich bereits die Spuren seiner Wende zum Christentum und zum Katholizismus. Aber diese Wende macht es noch nicht zu einem katholischen Buch, ist doch das originelle Werk mit seiner frühen Philosophie, vor allem der Evolution Creatrice (1907), die ihn unter katholischen Denkern verdächtig gemacht hatte, tief verbunden. Die zentrale Kategorie bei Bergson ist nicht der Glaube, sondern die subjektive Erfahrung der Mystik. Die Religion wird wesentlich von der Mystik her verstanden, deren Höhepunkt für Bergson die christlichkatholische Mystik ist. Die mystische Erfahrungsdimension ist für Bergson in mehrfacher Hinsicht von großer Bedeutung, einerseits für seine eigene Philosophie, andererseits für eine neue Behandlung der klassischen Fragen der

6 Kolakowski 1985, p. viII. 
philosophischen Theologie. Die Betrachtung der Religion aus der Perspektive der Mystik steht im Kontext von Bergsons Ausbildung in der religionsund mystikkritischen Psychologie seiner Zeit, die er mit Hilfe eines neuen Studiums der Mystik zu erweitern suchte. Am Schluss des Buches entwickelt er mit Hilfe dieser Psychologie eine neue Lehre des Menschen im technischen Zeitalter.

Naturphilosophie und Psychologie

Die Psychologie Bergsons setzt bei der Zeitphilosophie an, die ihn philosophisch bekannt gemacht hat. Wir gehen hier kurz, viel zu kurz, darauf ein, um den Hintergrund seiner Religionsphilosophie zu beleuchten. ${ }^{7}$ Bergson unterscheidet zwischen "gelebter Zeit" (durée) und "räumlicher Zeit" (temps d'espace). Die gelebte Zeit ist ein Kontinuum, das, sich sammelnd, in der Erinnerung anwesend ist. „Temps d'espace“ ist hingegen die Zeit, die in gleichen Zahlelementen, quasi räumlich verteilt, anwesend ist. Diese Zeitphilosophie hat in der Ästhetik zur Bestimmung der musikalischen Zeit sowie in der Filmästhetik von Deleuze weitergewirkt. Nach Bergson ist die Zeit schöpferisch. Zeit ist, wie er noch vor Heidegger betonte, das eigentliche Sein. Sein revolutionäres Ziel war es, den statischen Seinsbegriff der traditionellen Philosophie mit Hilfe seiner Zeitphilosophie durch eine Philosophie des Werdens zu ersetzen. Die dynamische Naturphilosophie ist von der Zeit, d.h. von der Psychologie her gedacht. Die Natur ist nicht als solche der Raum des räumlichen Zeiterlebnisses. Vielmehr ist es unser räumliches Zeiterlebnis, das die Natur zum statischen Raum macht; die Natur an sich ist dynamisch-zeitlich und wird von einem schöpferischen, zeitlichen, auch als göttlich verstandenen Trieb, dem élan vital, regiert, der als göttliche Psyche gedacht werden muss. Die Seele oder der Geist äußern sich schöpferisch in den verschiedenen Dimensionen der Natur, die nicht aufeinander reduzierbar sind. Die Natur ist ein Universum mit Brüchen oder Sprüngen, auch innerhalb des Menschen in seiner Dualität von Natur- und Kulturwesen. Der Mensch ist immer beides und immer im Übergang. Seine Wirklichkeit, zum Beispiel seine Gesellschaftlichkeit und seine Religion, ist sowohl auf der natürlichen als auch kulturellen Ebene präsent.

7 Für diese Übersicht verwenden wir Kolakowski 1985. 
Die Anfänge von Bergsons Denken liegen in der Psychologie. Seine erste wissenschaftliche Publikation, 1886 in der Revue Philosophique publiziert, handelt von der „Unbewussten Simulation im Zustand der Hypnose“ (Simulation inconsciente dans l'état de hypnotisme), noch bevor Breuer und Freud darüber geschrieben haben. 1913 wurde er Vorsitzender der „Society for Psychical Research“ in London. Bergson kannte Freud, war aber selber kein Psychoanalytiker; 8 er erwähnt ihn in verschiedenen Werken, jedoch nicht in Les deux Sources.

In der Psychologie seiner Zeit wurde Religion als eine Krankheitserscheinung betrachtet. Religion war ein Fall der Hysterie und der Halluzination. Für Pierre Janet, der auch Freud bekannt war, galt die Mystik als Höhepunkt bzw. als die eigentliche Erscheinungsweise dieser Krankheit. In seiner Vorlesung Une extatique mystique (Eine mystische Ekstatikerin) spricht Janet über die Krankheit einer Patientin und über ihre mystischen Erfahrungen: diese Analyse wurde bezeichnenderweise in der Zeitschrift Histoire de la folie (Geschichte des Wahnsinns) publiziert. Janets großes Werk De l'angoisse a l'extase (Von der Angst zur Ekstase) hat den Untertitel Une délire religieux chez une extatique (Ein religiöses Delirium bei einer Ekstatikerin). In kritischer Absetzung von Janet ${ }^{9}$ löst Bergson das Studium der Mystik aus dem Kontext positivistischer Psychologie, die ihm inhaltlich und methodisch als unzureichend erscheint. Dabei ist Bergson auch von William James inspiriert, der ebenfalls einen positiven Zugang zu Religion und Mystik sucht. Bergson distanziert sich jedoch nicht von der psychologischen Betrachtung der Religion als solcher, sondern zielt auf eine neue Psychologie, die die menschliche Seele auch in ihrer Tiefendimension versteht.

Damit kommen wir zu Bergsons eigener, d.h. aus seiner eigenen Philosophie herrührenden Motivation für eine Auseinandersetzung mit Religion und Mystik. Bergsons Philosophie ist eine Philosophie der Intuition. ${ }^{10}$ Dass die Vernunft auf eine nichtsinnliche Schau hin ausgerichtet ist, ist eine alte Weisheit der griechischen, genauer der (neu)platonischen Philosophie. In der modernen Philosophie von Descartes bis Kant und Hegel kehrt das Thema der Intuition unter dem Titel „intellektuelle Anschauung“ in der Bestimmung des Verhältnisses zwischen Verstandeserkenntnis und sinnlicher Anschauung wieder. Da für endliche Vernunft nach Kant eine intellektuelle Anschauung

\footnotetext{
8 Siehe Lawlor/Moulard 2016.

9 Bergson 1970, Kap. III,5.

10 Kolakowski 1985, pp. $24 \mathrm{ff}$.
} 
unmöglich ist, setzt sich Bergson in L'Evolution creatrice und in La Pensée et le Mouvant ausführlich mit Kants Erkenntnistheorie auseinander. Bergsons Begriff der Intuition ist jedoch breiter als der klassische Intuitionsbegriff. Der Begriff der Intuition deckt bei Bergson verschiedene Dimensionen ab, z.B. die Empathie, das Miterfahren des Ganzen in einer Teilerfahrung, aber auch die konnaturale, d.h. aus persönlicher Verwandtschaft und Zuneigung stammende Erkenntnis und nicht zuletzt die mystische religiöse Schau, die für uns hier wichtig ist, denn nach Bergson gibt es eine Intuition des kosmischen Lebensschwungs, des élan vital im Kosmos, der das schöpferische Vermögen Gottes oder Gott selber ist (Bergson 1970, Kap. II,4; III,1, 2). Die Vernunft als solche, d.h. die pragmatisch-technische Rationalität, die in einer sachlichen Nähe zum pragmatistischen Vernunftbegriff von William James steht, besitzt hingegen nach Bergson kein schauendes Vermögen. Der Bezug zu den Sachen selbst ist allein Sache der ästhetischen und der religiös-mystischen Intuition, nicht der Vernunft als solcher. Die mystische Intuition ist nach Bergson nicht bloß eine theoretische Haltung, sondern - vor allem im Christentum - stets mit Aktion und Liebe verbunden. Aus diesem Grund leitet die Mystik die Menschen zum Handeln an, was die Vernunft als solche nicht vermag, weil sie nur durch einen Anstoß von außen in das Handeln übergehen kann. Religion ist daher nach Bergson zuallererst als Handlung zu verstehen. In der Verbindung der Intuition mit der Praxis liegt nach Bergson auch der Unterschied zu Plotins quasi-mystischer Schau.

Da die Religionspsychologie einen großen Einfluss auf die zeitgenössische Kulturanthropologie ausübte, setzt sich Bergson auch mit Theorien auseinander, die, wie z.B. bei Lévy-Bruhl, die Religion zwar nicht als Krankheit, aber als prälogische Geisteshaltung qualifizieren (Bergson 1970, Kap., III,1, 2, 3, 12). Lévy-Bruhl verwendet für den prälogischen Status der Religion auch den Begriff Participation mystique (mystische Teilnahme), die das Merkmal einer mentalité primitive (primitiven Mentalität) sei. Da der primitive Mensch keine sekundären Ursachen anerkennt, sucht er nach mystischen Ursachen. Die primitive Vorstellungswelt, die das Gesetz des Widerspruchs noch nicht kennt, lebt in einer mystischen Teilnahme an Allem, ohne Bewusstsein der Subjekt-Objekt-Relation. Da die mystische Teilnahme allen Religionen gemeinsam ist, ist nach Lévi-Bruhl Religion insgesamt ein primitives Phänomen. Zwischen Lévy-Bruhl undJanet entstand eine Diskussion über den kollektiven oder individuellen Charakter religiöser Vorstellungen. In dieser Diskussion zwischen Psychologie und Soziologie (besonders Durkheim) schlägt Bergson ein alternatives Verhältnis zwischen primitiven Religionen und Weltreligionen vor. Bergson unterscheidet zwischen der Religion einer geschlossenen und einer offenen Gesellschaft, der die Differenzierung zwischen 
statischer und dynamischer Religion korrespondiert. Auch die primitiven Religionen können daher nicht einfach als irrational disqualifiziert werden. Die Mystik hingegen steht an der Quelle der offenen Gesellschaft und der dynamischen Religion.

\section{Geschlossene und offene Gesellschaft}

Bergson beginnt in Les deux sources mit einer Beschreibung der geschlossenen Gesellschaft, die sich eng an die Begriffswelt von Durkheim anschließt. Durkheims soziologische Frage ist: Warum tun Menschen, was sie tun sollen? Die Ausübung der Moral entspringt nach Durkheim nicht einfach aus einem reinen Pflichtgefühl, sondern aus einem Druck der Gesellschaft. Die Moral dient der Selbstbewahrung und Kohäsion der Gesellschaft. Religion hingegen ist bloß der Ausdruck bzw. die Symbolisierung der Moral, der jeweils geltenden Werte der Gesellschaft. In religiösen Ritualen feiert die Gesellschaft sich selbst; sie zelebriert sich vor den Augen ihrer Mitglieder als Vorbild und erneuert sich so in den Herzen der Gläubigen.

Durkheim orientierte sich in seiner Deutung der Religion an den primitiven Religionen bzw. Gesellschaften, in denen noch die ursprüngliche Essenz der Religion greifbar sei. Nach Bergson hingegen bilden die primitiven Religionen bloß ein bestimmtes, notwendiges Stadium in der Geschichte der Religionen und Gesellschaften, nämlich das Stadium des Organismus, in dem der religiösgesellschaftliche Zusammenhang durch Gewohnheit gebildet wird. In der Gesellschaft als Organismus ist für einen Individualismus noch kein Platz (Bergson 1970, Kap. I,2.3). Die Moral dieser Gesellschaft manifestiert sich in Gewohnheiten und dient der Selbstbestätigung der Gesellschaft. Die Macht der Gewohnheiten erscheint wie ein Naturgesetz, das von den Religionen z.B. mit dem Begriff der Schöpfung narrativ dargestellt wird. Die geschlossene Gesellschaft ist die erste „natürliche“ Gestalt der Gesellschaft und der Moral überhaupt, die selber keine natürlichen Entitäten sind, sondern etwas Neues, eine Neuschöpfung im Kosmos. In menschlicher Hinsicht ist die „organische“ Gesellschaftsformation jedoch die erste „natürliche“ Form der Gesellschaft. Wesentlich für geschlossene Gesellschaften ist weiters die Abgrenzung von anderen Gesellschaften, genauer eine Selbstbehauptung durch Ausschließung anderer. Der Horizont der Menschheit als solcher ist in geschlossenen Gesellschaften noch nicht präsent. In der modernen Zeit ist z.B. der Nationalismus Ausdruck einer geschlossenen Gesellschaft.

Die offene Gesellschaft, die die Menschheit selbst ins Blickfeld rückt, ist eine schöpferische Neubildung, sie markiert einen Sprung in der kulturellen 
Entwicklung, in der mit Gott zugleich die Menschheit entdeckt wird. Die Entstehung der offenen Gesellschaft ergibt sich nicht aus einem graduellen Übergang von der Familie zur Gesellschaft und schließlich zur Menschheit. Die Entdeckung der Menschheit ist vielmehr eine gesellschaftliche Revolution, die auch eine Revolution der Moral miteinschließt. Der Sprung zu einer offenen Gesellschaft manifestiert sich nach Bergson in großen Persönlichkeiten, konkret in den Heiligen bzw. Mystikern des Christentums, den Weisen Griechenlands, den Propheten Israels und den mystischen Arhats des Buddhismus (Bergson 1970, Kap. I,6). Diese Figuren, die Jaspers historisch in der Achsenzeit um 500 vor Chr. situiert, werden von Bergson im Lichte der Mystik gedeutet, die am vollkommensten in der christlichen Mystik ausgeprägt ist.

Die neue Moral stützt sich nicht auf sozialen Druck oder Gewohnheit, sondern auf Vorbild und den Appell zur Nachfolge. Der mystische Appell ist eine Einheit von Vernunft, Emotion und Praxis - Charité, amour de l'humanité (Nächstenliebe und Menschheitsliebe). Im Neuen Testament wird diese Moral vor allem in der Bergpredigt formuliert, die nach Bergson unabhängig davon, ob man Jesus als Gott oder Mensch betrachtet, gültig ist. Für Bergson ist die Frage, ob Jesus Gott oder Mensch ist, sekundär, da er von der Göttlichkeit aller Menschen ausgeht (Bergson 1970, Kap. III,6). Bergson bewegt sich hier in einer spezifischen Strömung seiner Zeit. Bereits Tolstoi hatte die Bergpredigt wiederentdeckt und auf diese Weise indische Denker, die auch Bergson bekannt waren, inspiriert. Aus dieser Moral folgen nach Bergson letztlich auch die Menschenrechte. Für die Idee der Gerechtigkeit in einer offenen Gesellschaft sind die Propheten Israels von eminenter Bedeutung. Die Propheten stehen gleichsam an der Schwelle der geschlossenen Gesellschaft des jüdischen Volkes zur offenen Gesellschaft, da sie in ihrer Botschaft an das Volk Gottes zugleich den Blick auf die gesamte Menschheit ausweiten. Die Botschaft universeller Gerechtigkeit wird jedoch nach Bergson erst durch das Christentum, das das Ideal der Gerechtigkeit in seine Mystik integriert, vollendet. Das Judentum kennt als "Schwellenland“ nach Bergson keine Mystik; sein Spezifikum ist die Idee der Gerechtigkeit (Bergson 1970, III,5, 6 (7)). Bergson blendet daher die jüdische Mystik (Kabbala, Chassidismus) aus. Die Unterscheidung zwischen prophetischer und mystischer Religion, die für Bergson offenbar eine wichtige Rolle spielt, ist noch jung und wurde erst im frühen 20. Jahrhundert von den Religionswissenschaftlern Rudolf Otto und Nathan Söderblom eingeführt. Bergson rezipierte Söderblom auch für seine Interpretation des Buddhismus. ${ }^{11}$

11 Vgl. dazu Antes 1998. Für die Erwähnung Söderbloms als „Religionshistoriker“ siehe Bergson 1970, Kap. III,4 und die „Notes Historiques“, p. 1571; p. 1166. 
Die Unterscheidung zwischen offener und geschlossener Gesellschaft hat verschiedene Ebenen, die nicht klar differenziert sind. Einerseits scheint es sich um zwei Typen von Gesellschaft zu handeln, die grundsätzlich zu unterscheiden sind; anderseits scheint es um ein Verhältnis von Ideal und Realität bzw. um zwei Dimensionen ein und derselben Gesellschaft zu gehen. Zudem sollen nach Bergson die Menschenrechte der offenen Gesellschaft wie auch andere moralische Haltungen und Prinzipien natürlich auch zur Gewohnheit werden, womit sie die Formen der geschlossenen Gesellschaft für ihre eigene Realisierung brauchen (Bergson 1970, Kap. IV,1,2).

Die geschlossene Gesellschaft hat eine statische Religion. Im Kontext der Theorie der offenen Gesellschaft entwirft Bergson - in kritischer Auseinandersetzung mit Durkheim und Lévi-Brühl - eine eigene Theorie hinsichtlich der Funktion der Religion in der Gesellschaft, worauf ich nur kurz eingehen möchte. Bergson verfolgt nicht die Strategie, die Kritik der Religion allein auf die primitiven Religionen zu beschränken, um zu Janet und anderen gleichsam sagen zu können: „Ihr habt recht mit eurer Kritik, aber sie stimmt nur für die Primitivreligionen." Bergson entwickelt vielmehr eine neue Deutung des Mythos, des Totemismus und anderer Phänomene, die in seiner Zeit im Licht des Schemas „Rationalität und Irrationalität" gedeutet wurden. Nach Bergson haben die Primitiven keine andere Mentalität als wir.

Die Mythen entspringen nicht der Irrationalität; sie dürfen auch nicht als noch primitive Weisen des Wissenschaftstreibens verstanden werden. Mythen entstammen der "fonction fabulatrice“ (Fabulierfunktion) bzw. der „storytelling, myth-making function“ (Bergson 1970, Kap. II,2). „Fabula“ ist das lateinische Wort für Mythos, der eine Fiktion ist. Im wissenschaftlichen Sinne sind mythisch-religiöse Geschichten nicht wahr; sie beanspruchen auch nicht, eine „alternative“ wissenschaftliche Erklärung anzubieten. Mythen über die Phänomene der Natur mit den entsprechenden Geistern und Göttern sind keine alternativen wissenschaftlichen oder kausalen Erklärungen, sondern sprechen über die Bedeutung der Natur und ihrer Erscheinungen für den Menschen. Mythen sind Sinngebungsgeschichten und haben daher eher mit der Finalität der Natur zu tun. Als Fiktionen sind sie mit der Kunst verwandt; daher werden die Mythen später zu ästhetischen Erzählungen. Durch Mythen stellt der Mensch sein Verhältnis zur Wirklichkeit, das durch die analytische Rationalität gebrochen ist, wieder her; sie sind kein Ersatz, sondern ein notwendiges Komplement zur Wissenschaft. Durch die analytische Ratio fällt die 
Welt auseinander; erst die Fabulierfunktion, die ein Werk der Natur, des élan vital, im Menschen ist, stellt das Ganze wieder her. Die Religion ist folglich für Bergson eine sich im Menschen ereignende Reaktion der Natur auf die zersplitternde Kraft des Denkens, die ebenfalls aus ihr stammt. Kurz: Religion ist ein Gegengewicht gegen die Instabilität, die wiederum eine Folge des Verschwindens des Instinkts ist. Religion ist daher ein Mittel gegen die Depression, die in der Sterblichkeit ihre Ursache hat. Religion entspringt nicht der Furcht, sondern ist eher eine Reaktion gegen die Furcht, vor allem gegen die Furcht vor dem Tod und vor der Wandelbarkeit des Lebens. Religion ist auch keine mystische Teilhabe oder participation mystique. Da die statische Religion über das Stadium der primitiven Religionen hinaus fortwirkt, unterscheidet sich die Intelligenz der Primitiven nicht von unserer Intelligenz. Allerdings gibt es in den primitiven und den statischen Religionen nach Bergson noch keine Mystik, die ausschließlich der dynamischen Religion zugeordnet wird. Im Verhältnis zur wissenschaftlichen Rationalität ist nach Bergson die statische Religion infrarational, die dynamische suprarational. Die dynamische Religion fällt im Grunde mit der Mystik zusammen. Mystik ist die Erfahrung der Identität mit Gott, d.h. mit der schöpferischen Kraft im Universum, die Gott selber ist (Bergson 1970, Kap. III,3).

Bergson beharrt in seiner Theorie der Mystik auf der Verbindung zwischen Theorie und Praxis, konkret der Caritas, der Liebe. Religion, die immer mit der Mystik verbunden ist, ist Tat. Die „reine“ Philosophie verfehlt die praktische Dimension der Religion, die letztlich Tat ist. Sokrates kommt durch seine Beziehung zu seinem "Daimonion“ noch am ehesten in die Nähe der praktischen Dimension der Mystik. Auch Plotins Denken entspringt einer religiösen Inspiration; durch den Einfluss des Ostens bleibt seine Mystik jedoch nach Bergson zu theoretisch. Erst in der christlichen Mystik trete die Einheit von Theorie und Praxis in aller Deutlichkeit hervor.

In der Religion gewinnt die Mystik darüber hinaus wieder eine gesellschaftliche Form, denn die Religion ist nach Bergson nichts anderes als die popularisierte Form der Mystik. Daher bleibt eine gewisse Verwandtschaft zwischen dynamischer und statischer Religion bestehen. So ist z.B. das Gebet nach Bergson in der dynamischen Religion formlos, insofern es als Bewegung der Seele seinen verbalen Ausdruck übersteigt; doch das Gebet ist nicht ohne eine Beziehung zu den Formen der statischen Religion (Bergson 1970, II,24). Die dynamische Religion verhält sich zur statischen Religion so, wie die offene zur geschlossenen Gesellschaft. Einerseits handelt es sich um zwei Typen von Religion, andererseits um zwei Dimensionen innerhalb einer Religion. In ihrer dynamischen Form ist die Religion per definitionem Weltreligion, was in praktischer Hinsicht heißt: Wer versteht, was Gott bedeutet, versteht 
zur gleichen Zeit die Menschheit und weiß unmittelbar, dass es keinen Krieg geben darf. Nächstenliebe, Gottgläubigkeit und Menschheitsglaube bilden nach Bergson eine untrennbare Einheit.

Um der Einheit von Theorie und Praxis willen erhebt Bergson die christliche Mystik zur Norm und zum Urbild aller Mystik. Als praktische Caritas steht die Mystik nicht im Gegensatz zur Idee der Verbesserung der Welt durch Technik und Industrialisierung, vielmehr versucht Bergson die Technik mit der Mystik $\mathrm{zu}$ verbinden. Mit seiner positiven Haltung zur modernen Technik reagiert er auf die Ideen des indischen Philosophen Swami Vivekananda, der durch seinen Auftritt auf dem Weltkongress der Religionen in Chicago 1893 weltberühmt geworden war. William James, ein Freund Bergsons, hatte Vivekananda in Amerika empfangen. Auf dem Kongress in Chicago traf Vivekananda die äußerst einflussreiche Unterscheidung zwischen dem mystischen Osten, vor allem in Indien, und dem technischen Westen.

Diese schroffe Gegenüberstellung ist nicht nur in Indien selbst beliebt geworden, um sich vom Westen zu unterscheiden, sondern auch unter westlichen Romantikern, Theosophen und im Umfeld anderer alternativer Weltanschauungen. Bergson jedoch weist Vivekanandas Unterscheidung zurück. Ramakrishna und Vivekananda können, so kontert Bergson, so leidenschaftlich mystisch sein, weil sich die Lebensumstände in Indien durch die Übernahme westlicher Wissenschaft und Technik bereits verbessert haben. Die alte indische Mystik ist nach Bergson theoretisch geblieben, da sie sich gegenüber der Armut als ohnmächtig erwiesen hat. Bergson legt hier den Finger auf einen neuralgischen Punkt im Dialog oder im Streitgespräch zwischen Ost und West. Reformhindus wie Vivekananda fühlten sich nämlich vom europäischen Vorsprung in der Wissenschaft und der aktiven christlichen Caritas mit ihren Schulen und Krankenhäusern einerseits angesprochen und herausgefordert, andererseits aber auch in ihrer kulturellen Identität bedroht. Vivekananda schätzt zwar die Praxis von Jesus, sieht in ihr jedoch zugleich seine Beschränkung gegenüber der Mystik des Ostens. Wie später Gandhi, so fürchtete auch Vivekananda, dass die christliche aktive Caritas zu einem Proselytismus führe. Bergson dreht hingegen den angeblichen Gegensatz zwischen spirituellem Osten und westlicher Technik um: Das Christentum, die christliche Mystik, ist wesentlich aktiv und geht darum in technische Weltverbesserung über. 
Der Yoga des Hinduismus ist nach Bergson noch keine Mystik, sondern nur ein Gefäß für die Mystik, die im Brahmanismus und Buddhismus präsent sei. Der Buddhismus hingegen, der auch Nächstenliebe und Mitleid kennt, komme der wahren Mystik zumindest nahe; Bergson vermisst hier aber noch die Wärme des Christentums (Bergson 1970, Kap. III,4.5). Die Mystik des Islams bleibt in seinem Werk bedauerlicherweise völlig ausgeblendet.

Die Theorie der christlichen Mystik als Einheit von Theorie und Praxis gibt Bergson auch in methodischer Hinsicht die Gelegenheit, die „krankheitspsychologische" Betrachtung der Mystik zu kritisieren und zu überwinden. Wie gewinnen wir einen Begriff der Mystik? Für die Bildung des Begriffs der Mystik müssen wir, wie Bergson im Anschluss an Evelyn Underhill und Henri Delacroix zu Recht betont, hermeneutisch von der faktischen Mystik und ihren großen Gestalten ausgehen und nicht von Patienten, bei denen mystikähnliche Zustände vermutet werden (Bergson 1970, II,4, Fn. 2). Die großen Mystiker warnten selbst vor pathologischen Zuständen, die auf dem mystischen Weg auftreten können. Die Ekstasen dürfen jedoch nach Bergson nicht auf solche pathologischen Zustände, Halluzinationen u.a. beschränkt werden; denn Ekstasen sind Umwälzungen in der Person, in denen sich der revolutionäre Übergang von der traditionellen Religion und Gesellschaft zur dynamischen Menschheitsreligion manifestiert. Man kann die Mystiker und ihre Zustände mit schöpferischen Künstlern vergleichen, die ebenfalls tiefe Krisen durchleiden, wenn sie am Übergang zu Neuem stehen. Die Erscheinungen, die die Psychologen untersuchen, sind nach Bergson „infrarational“, die Emotion der Mystik hingegen ist „suprarational“. Die Mystiker, die Bergson vor Augen hat, sind vor allem Paulus, Theresa von Avila, Katharina von Siena, Franziskus und die für einen Franzosen damals unvermeidliche Jeanne d'Arc.

Die existentielle Umwälzung führt am Ende zu einer großen Freude (joie), die vom bloßen Vergnügen (plaisir) zu unterscheiden ist. Diese Freude, die großartig und zugleich einfach ist, ist charakteristisch für die mystische Erfahrung, in der der Mensch fühlt, dass Gott in der Seele handelt, wodurch der élan vital neu zur Erscheinung kommt. In der mystischen Erfahrung liebt man die Menschheit in Gott und Gott in der Menschheit. Bergson legt immer Wert darauf, neue Phänomene nicht als graduelle Erweiterung älterer Formen der Religion und der Gesellschaft zu deuten. Die offene Gesellschaft verwirklicht sich vielmehr in einem Sprung über die geschlossene Gesellschaft 
hinaus. Im selben Sinn verhält sich die dynamische zur statischen Religion. Die Mystik als Einheit von Gottesglaube und Menschheitsbejahung ist auch keine Erweiterung des sozialen Instinktes, sondern etwas radikal Neues. Obwohl der Begriff der Mystik im Ausgang von den außerordentlichen Persönlichkeiten und ihren Erfahrungen entwickelt werden muss, ist die mystische Erfahrung keineswegs nur eine Sache privilegierter Persönlichkeiten. Zwar kann umgekehrt nicht behauptet werden, dass jeder ein Mystiker ist, die mystische Erfahrung, die zunächst von großen Gestalten durchlebt wird, kann jedoch von andern Menschen mit- und nacherlebt werden. So können wir alle an der mystischen Erfahrung teilhaben. Die Mystik ist entflammte Religion, die Religion hingegen ist eine Abkühlung der Mystik. So wie sich in der Alltagswelt wissenschaftliche Kenntnisse vulgarisieren, ist auch Religion eine Vulgarisierung der Mystik (Bergson 1970, II,6).

Durch das Nacherleben mystischer Erfahrungen ist auch eine Nachfolge möglich! Die Nachfolge vollzieht sich vor allem in der Arbeit der Orden. Die christlichen Mystiker selbst folgen Jesus nach, der für Bergson selbst als Mystiker und nicht als Sohn Gottes verstanden wird, denn, wie bereits erwähnt, sind für Bergson alle Menschen göttlich. Diese Aussage gewinnt am Ende des Buches, wo mit Hilfe der Mystik eine neue Gestalt des Menschseins entworfen wird, eine spezifische Relevanz.

\section{Mystik und die Erneuerung der philosophischen Theologie}

Bergson geht es in seinem Werk nicht nur um eine neue Religionspsychologie, sondern auch um die Erneuerung der klassischen philosophischen Theologie (Bergson 1970, Kap. III,8-13). Mit der Mystik bringt er die Dimension der Erfahrung in die begriffliche Welt der philosophischen Theologie ein. In den traditionellen Gottesbeweisen haben die Philosophen nach Bergson Gott jeweils falsch definiert, nämlich als eine die Erfahrung übersteigende Entität, die keine wirkliche Beziehung zum Menschen hat. Ein solcher Gott hat mit dem Gott der Religion, sei es der griechischen Mythologie oder des Christentums, nichts zu tun. In der Religion gilt, dass Gott in eine Beziehung zu den Menschen tritt, was in der mystischen Erfahrung gleichsam „verifiziert“ wird: In der Mystik "beweisen“ die Religionen, dass Gott erfahren wird. Dieses Argument stützt sich auf die Konvergenz zwischen den verschiedenen Mystikern, die letztlich alle dieselbe Entwicklung durchlaufen würden. Zwar hat es manchmal den Anschein, als ob Bergson einen Gottesbeweis aus der Übereinstimmung zwischen den Mystikern, ex consensu mysticorum, herleiten möchte, doch geht er nicht so weit. Für ihn ist die mystische Erfahrung eine 
zuverlässige Erfahrung. Aus der Perspektive der Wissenschaft könnte man einwenden, dass Erfahrungen keinen wissenschaftlichen Wert haben, solange sie nicht von allen und für alle wiederholt werden können. Aber wer, so repliziert Bergson, kann schon ein wissenschaftliches Experiment wiederholen? Dazu kommt, dass jeder Mensch in sich einen Widerhall dieser Mystik fühlen kann. Bergson beruft sich an dieser Stelle auch auf William James, der eingestand, nicht selbst Ekstasen erfahren zu haben, jedoch ihren Widerhall zu fühlen. In potentia sind wir nach Bergson alle Mystiker. Die mystische Erfahrung wird dabei nicht als neuer Gottesbeweis eingeführt. Bergson möchte vielmehr den religiösen Gott wieder ins Bewusstsein heben, ohne sich auf eine bestimmte Religion oder Offenbarung zu berufen, obwohl er die christliche bzw. katholische Mystik als „die“ Mystik ansieht. Es geht ihm um den religiösen Gott, wie er in der menschlichen Erfahrung erscheint. Trotz aller Differenzen zur Philosophie konvergiert die mystische Erfahrung mit philosophischen Überlegungen zu Gott als unendlicher Kreativität. Die Wissenschaft aber akzeptiere nach Bergson auch ein Argument aus der Konvergenz verschiedener Tendenzen.

Sehr wichtig für Bergson ist weiters, dass die Mystik die wechselseitige Beziehung zwischen Gott und Mensch, Schöpfer und Geschöpf aufweist. Gott ist auf der Suche nach dem Menschen, so wie der Mensch auf der Suche nach Gott ist. Bergsons lebensphilosophischer Begriff der Relationalität Gottes bezeugt seine durchgängige schöpferische Anwesenheit in allen Dingen. Im Gegensatz zum unwandelbaren aristotelischen Gott, den Bergson als einen nichtreligiösen Gott ansieht, ist von der Mystik her die Gottesbeziehung als eine Liebesbeziehung zu verstehen. Am Ende der schöpferischen Liebesbewegung im Kosmos steht der Mensch selbst als Schöpfer. In der Gottesbeziehung stehen also der göttliche Schöpfer und der menschliche Schöpfer in einer Liebesbeziehung zueinander. Der späte Bergson überdenkt seine gesamte Lebensphilosophie und ihre Prinzipien im Lichte der mystischen Erfahrung und umgekehrt. Wir können darauf nicht weiter eingehen. Ich möchte hier nur noch auf zwei Themen der philosophischen Theologie kurz hinweisen, die Theodizee und die Unsterblichkeit.

Obwohl seine Lebensphilosophie grundsätzlich optimistisch ist, weiß Bergson genau, dass man das Übel aus der Welt nicht eliminieren kann. Er wendet sich zunächst dem Leid als Schmerz und dem Schmerzbewusstsein der Tiere zu. In der Folge werden die verschiedenen Arten des menschlichen Leidens, ob verschuldet oder unverschuldet, behandelt. Die Theodizee von Leibniz prallt nach Bergson an den konkreten Leiderfahrungen ab. Das Leid einer Mutter, die ihr Kind sterben sieht, bleibt eine schreckliche Realität. Zugleich ist nach Bergson ein bestimmter Optimismus gerechtfertigt, obwohl er 
nichts erklärt. Erstens ist es eine Erfahrung, dass das Leben im Ganzen gut ist und dass Menschen darum am Leben hängen. Zweitens gibt es die mystische Erfahrung, die eine positive Erfahrung der Fülle ist, die Erfahrung einer Freude jenseits von Lust und Schmerz. Beide Erfahrungen sind eine Bejahung des Guten inmitten von Leid.

Die mystische Erfahrung gibt auch der Hoffnung auf ein Leben nach dem Tode einen Inhalt. Die klassische platonische Definition der Seele und ihrer Unsterblichkeit ist wegen ihrer reinen Begrifflichkeit unfruchtbar. Auch in der schwierigen Frage nach dem Jenseits ist die Erfahrung nicht bedeutungslos. Im Gegenteil: Was wir aus der wissenschaftlichen Erfahrung kennen, ist die Unzulänglichkeit der physiologischen Erklärungen des Gedächtnisses. Da wir hier und jetzt schon die Erfahrung der Unabhängigkeit seelischer Zustände von der Körperlichkeit haben, können wir möglicherweise auf ein Fortleben der Seele nach dem Tod schließen. Aber auf welches Wissen können wir uns hier stützen? Wird das Fortleben für eine gewisse Zeit sein oder für ewig?

Diese Erfahrung „von unten“ soll durch die Erfahrung „von oben“, die mystische Intuition, ergänzt werden. Ist das Fortleben der Seele identisch mit jenem Fortleben, in das bevorzugte Seelen schon hier unten eingehen dürfen? Mit den Untersuchungen über dieses Verhältnis wird das Problem zwar nicht einfach gelöst, aber doch eine fruchtbare Perspektive eröffnet. Bergson ist zu Recht vorsichtig. In existentieller Hinsicht ist die inhaltliche Deutung der Idee der Unsterblichkeit der Seele mittels der mystischen Erfahrung natürlich berechtigt. Mit dieser Deutung kommt zum Ausdruck, dass die Beziehung zwischen der Seele und Gott auch der Erfahrung des Todes standhält. Die Hoffnung auf ein Leben nach dem Tod ist eine eindrucksvolle Glaubensaussage über das Gottesverhältnis, das der Mensch in sich fühlt. Zahlreiche Mystiker haben ihre ekstatischen Intuitionen als einen Vorgeschmack bzw. als Antizipation der ewigen Schau von Angesicht zu Angesicht in der Ewigkeit verstanden. ${ }^{12}$ Bergson hingegen lotet die Möglichkeiten aus, ob die mystische Erfahrung unsere Erkenntnisse über ein Weiterleben der Seele erweitern könne. Unübersehbar ist Bergsons Intention, den alten Gegensatz zwischen einer materialistischen und einer spiritualistischen Auffassung der Seele zu überwinden; zugleich ist jedoch mehr als deutlich, wie vorsichtig Bergson in dieser Frage vorgehen will. Aber die Hoffnung bleibt lebendig und kehrt in einem anderen Kontext am Ende des Buches wieder zurück. 
Im Schlusskapitel wendet sich Bergson aktuellen Problemen seiner Zeit zu: dem Weltfrieden, der Stiftung des Völkerbundes und der modernen Technik. Auch Jaspers thematisierte in Ursprung und Ziel der Geschichte diese Themen im Lichte der Gründung der Vereinten Nationen. Der Inhalt eines Dialogs der Religionen und der Philosophien sollte nach Jaspers die Beherrschung der Technik sein. Dieser Themenkomplex kündigt sich bereits bei Bergson an, wenn auch auf eine andere Weise. Jaspers steht als Existenzphilosoph der Technik weitaus kritischer gegenüber als Bergson, der in seinem evolutionären Denken ihr gegenüber eine optimistische Haltung einnimmt.

Die Probleme des Weltfriedens und der Technik sind nach Bergson aufs Engste mit der Aufgabe der Bewältigung der Probleme der industriellen Gesellschaft verbunden, denn mit der industriellen Gesellschaftsform werden die ökonomischen Gründe des Krieges vorrangig. Bergson entwickelt im letzten Kapitel seines Werkes zahlreiche Gedanken über die Beziehungen zwischen Krieg und menschlicher Natur in der Geschichte und den jeweils korrespondierenden Gesellschafsformen. Wir beschränken uns hier auf Bergsons Gedanken über Krieg in der modernen Gesellschaft, in der die Gründe für den Krieg zumeist wirtschaftlicher Natur sind. Die alten Kriegsideen der großen Eroberer, aber auch die ritterliche Idee des Krieges sind längst überholt. Damit ist der Krieg als solcher nicht vorbei, er gehört zur Natur des Menschen, da in der geschlossenen Gesellschaft der Gegensatz zwischen dem „Wir" und „den Anderen“ wesentlich ist. In der Moderne sind die zentralen Gründe für Krieg hingegen Bevölkerungszunahme, Wachstum der Produktion, Suche nach neuen Märkten, Gewinnung von Rohstoffen usw. Bergson hofft, dass in Zukunft diese Kriegsgründe vom Völkerbund beherrscht werden können.

Die Technik ist mit all diesen Problemen sachlich verbunden, weil sie ein Wachstum der Produktion ermöglicht und gleichzeitig neue Bedürfnisse schafft. Im Unterschied zu Herbert Spencer, der in Bergsons Denken im Hintergrund stets präsent ist, geht Letzterer nicht davon aus, dass industrielle Gesellschaften wesentlich friedsame Gesellschaften sind. Der Kriegsinstinkt bleibt auch in modernen Gesellschaften lebendig. Allerdings sind die offene Gesellschaft und die dynamische Religion, die als Mystik verstanden wird, dem kriegstreibenden „Ingroup-outgroup“-Denken diametral entgegengesetzt. Allerdings spürt Bergson in den Theorien und Publikationen seiner Zeit die Vereinnahmung der Mystik durch das nationalistische Denken, das sich selbst eine göttliche Aufgabe in der Welt zuschreibt. Imperialismus verschmilzt so mit einem bestimmten Mystizismus. Das aktuelle Phänomen einer nationalistischen oder imperialistischen "Mystik“ kann nach Bergson 
nicht mehr geleugnet werden. Dennoch weist er beharrlich darauf hin, dass dabei das Wort "Mystik“ im Gegensatz zu seiner ursprünglichen Bedeutung verwendet wird. Eine imperialistische Welteinheit als mystische Idee ist für Bergson geradezu eine Parodie auf die wahre Mystik.

In der Frage nach der Beherrschung der Technik macht Bergson einige überraschende Bemerkungen, die sich nicht mehr unmittelbar auf die Problematik des Weltfriedens beziehen, jedoch am Ende wichtig werden. Die erste Überlegung knüpft an eine Diagnose von Rousseau an, die in der Folge für die politische Philosophie von Kant, Hegel und Marx eine große Herausforderung darstellte: Da der technische Fortschritt immer wieder neue, „künstliche“ Bedürfnisse schafft, scheint die Lösung des Problems des Weltfriedens darin zu liegen, den Prozess der künstlichen Vermehrung der Bedürfnisse zu beherrschen. Bergson prüft hier die Möglichkeit, ob die Mystik in der klassischen Verbindung mit der Askese ein Mittel zur Bewältigung dieser Art des Fortschritts sein könnte. Für Bergson ist die Kombination von Mystik und Askese Sache einer Elite. Aber Bergson möchte auch eine Mystik für alle, die vor allem in der Caritas, d.h. der Hilfe für Menschen, die nichts zu essen haben, besteht. Die Technik gehört nach Bergson zur Caritas. Daher nimmt Bergson einen anderen Weg zur Bestimmung der Technik, der zu seiner schöpferischen Evolutionsphilosophie besser passt. „Künstlichkeit“ ist wesentlich menschlich und gut! Auf diese Weise schafft der Mensch etwas Neues, wodurch er die Natur übersteigt. Technik als Entwicklung der Kunst des Künstlichen heißt Entdeckung des Neuen, was wesentlich zum Menschen gehört. Dass Technik auch neue Bedürfnisse schafft, ist normal und gut; denn so entdeckt der Mensch in sich selbst neue Dimensionen. Natürlich können technische Entwicklungen auch schiefgehen; dennoch ist die Technik selbst wesentlich positiv. Auch die moderne Technik folgt aus der menschlichen Natur; ihre Verbreitung in der Form der industriellen Gesellschaft gehört zur Nächstenliebe. Die Mystik als Caritas hat nach Bergson die Entwicklung der Technik stimuliert. Technik und Caritas sind, wie bereits erwähnt, in der Bekämpfung der Armut wesentlich miteinander verbunden. Darum gehören auch Mystik und Technik zusammen (Bergson 1970, Kap. IV,10).

Nach Bergson haben wir uns durch neue Techniken und ihre Produkte de facto einen neuen Leib gegeben. Damit haben wir in gewissem Sinne die Natur, auch unsere leibliche Natur, überlistet. Die Sinnesorgane funktionieren im menschlichen Leben pragmatisch und selektiv. Bergson hat eine Art FilterTheorie des Bewusstseins; die Organe beschränken das menschliche Bewusstsein und Interesse auf das, was Hier und Jetzt für das Fortleben wichtig ist; sie wehren daher Signale aus dem Jenseits ab. In der modernen Technik, in der neue Dimensionen und Energien der Natur freigesetzt werden, die die 
Natur von sich her nicht eröffnet hatte, ist jedoch diese angeborene Diesseitsbeschränkung bereits relativiert. Allerdings ist unsere Seele Bergson zufolge auf diese neue Situation noch nicht eingestellt. Weiters betrachtet er die Entwicklung der Technik aus der Sicht der Organverlängerung. Unsere Instrumente sind in gewissem Sinne künstliche Organe, das Werkzeug des Arbeiters ist eine Verlängerung seiner Arme. Jedoch haben wir in der modernen Technik, die ungeheure Kräfte entwickelt hat, unseren Leib so sehr vergrößert, dass wir uns gleichsam einen neuen Leib gegeben haben. Die Welt der Technik ist jetzt unser neuer Leib, aber dieser ist noch ohne innere Belebungskraft, ohne Seele, weil diese sich mit der Technik noch nicht in adäquater Weise mitentwickelt hat. Daher braucht der neue Leib eine neue Seele, und diese neue Seele soll nach Bergson die Mystik sein! Auf diese Weise transzendiert sich der Mensch selbst in ein neues Stadium der Evolution. Wenn die beschränkende Funktion der Sinnesorgane durch die künstliche Welt überstiegen werden kann, offenbaren sich möglicherweise auch tiefere Dimension des Seelenlebens. Mit Hilfe der Technik hat der Verstand, der von Bergson wie William James wesentlich pragmatisch gedacht wird, die Bindung an die Sinnlichkeit überstiegen, womit die Intuition des Menschen mehr Raum erhält.

Am Ende seines Werkes treten die spekulativ-evolutionistischen Dimensionen von Bergsons Denken unverhüllt hervor. Auch in diesen Reflexionen zeigt sich in Bergsons Interesse an der Mystik eine existentielle und eine experimental-wissenschaftliche Seite. Aus existentiell-religiöser Sicht ist es überzeugend, das mystische Selbstbewusstsein im Dienste einer neuen Seele, d.h. der Beseelung der technisch-industriellen Kultur, zu denken. Dies ist ein fruchtbarer Gedanke neben der asketisch-mystischen Denkweise der Beschränkung aller Bedürfnisse. Aber die Botschaft, die Bergson uns mit der neuen Mystik geben möchte, ist nicht so sehr ein existentieller Appell, im neuen Zeitalter stärker auf die Tiefendimension unseres Bewusstseins zu achten. Ihm geht es nicht um die persönliche und religiöse Entwicklung und Frömmigkeit, sondern um eine psychologische und anthropologische Diagnose. Jetzt, wenn die beschränkenden Funktionen der Sinnesorgane verschwunden sind, könnte ein neuer Typus des Bewusstseins, ein erweitertes Bewusstsein entstehen, wofür die Mystik wichtig ist. In Bergsons Wahrnehmungspsychologie gibt es viele Welten der Erfahrung, die nicht hochkommen können, weil sie von unseren Sinnen, die selektiv auf die praktische Daseinserhaltung hin orientiert sind, abgeschirmt werden. In diesem Kontext sind für Bergson auch parapsychologische Phänomene wichtig.

Bergson hatte, wie bereits in Matière et Memoire (1896) sichtbar ist, sein Leben lang Interesse an Telepathie; seiner Meinung nach gab es genügend konvergierende Beweise für die Möglichkeit von Telepathie. Auch in seinem 
Spätwerk über Moral und Religion bezieht er am Schluss derartige Phänomene mit ein (Bergson 1970, Kap. IV,12). Insofern Bergson in das Studium der Mystik auch „abnormale“ parapsychologische Phänomene integriert, könnte man sich aus traditioneller philosophischer und theologischer Sicht fragen, ob er am Ende nicht selbst die Mystik mit einer falschen Psychologie verbindet. Bergson würde diesen Einwand brüsk zurückweisen. Wie die Mystik, so sind auch solch abnormale Phänomene ein Grund für eine Änderung der psychologischen Wissenschaft. Als Psychologe stand Bergson in Kontakt mit Fachgenossen wie Janet, Freud und Jung, die an diesen Phänomenen zumindest interessiert waren. Auch in seiner Antrittsvorlesung als Vorsitzender der British Society for Psychical Research hat sich Bergson mit diesem Thema auseinandergesetzt. ${ }^{13}$ In diesen Kreisen war der Einfluss Darwins sehr stark, dessen Evolutionstheorie Fragen nach einem neuen Stadium in der Evolution provozierte. Warum sollte der Mensch das Endstadium der Evolution sein? Mit diesen Gedanken war auch die Möglichkeit eines neuen, höheren Bewusstseins verbunden. ${ }^{14}$

Was Bergson als Resultat vor Augen steht, ist die Explikation der intuitivmystischen Dimension des Bewusstseins für die ganze Menschheit und ein erweiterter Begriff der (psychologischen) Wissenschaft, der auch diese Dimension erfasst. Auf diese Weise wird eine terra incognita entdeckt, die zu einer Umwälzung der Menschheit führt. Die Menschheit wird aus der Bindung an das Vorhandene befreit und empfänglich für die Botschaft des Jenseits, mit all den moralischen Aspekten, die zur dynamisch-mystischen Religion gehören. Im Gedanken, dass sich der Mensch in der Mystik und im modernen technischen Zeitalter nicht mehr auf diese oder jene kleinen Vergnügungen stürzen muss, kehrt bei Bergson schließlich auch die asketische Idee der Mystik wieder. Die Vergnügungen, die allesamt noch aus der Gebundenheit am Vorhandenen folgen, werden im Lichte der Ewigkeit verblassen und von der mystischen Freude überstrahlt werden. Die mystische Freude ist wesentlich mit der Einfachheit des Lebens verbunden.

Für diese epochale Umwälzung ist eine sittliche Reform notwendig, womit zuletzt auch die politische Dimension des technischen Fortschritts im Verhältnis zur Mystik zur Sprache kommt. So lange die radikale Reform aussteht, müssen wir uns mit dem Stückwerk der Politik und der Härte staatlicher Reglementierung zufriedengeben, um unsere Natur zu überwinden. Wie immer stellt der technische Fortschritt den Menschen im Großen und im Kleinen vor eine Entscheidung. Jetzt seufzt der Mensch unter dem Druck des

13 Barnard 2012, p. 288.

14 Diese Information verdanke ich dem Religionspsychologen Prof. Dr. Herman Westerink. 
selbstgeschaffenen Fortschritts, er soll jedoch nach Bergson verstehen, dass seine Zukunft von ihm selbst abhängt. Er soll sich entscheiden, ob er einfach so weiterleben will oder sich noch anstrengen möchte, damit sich auf unserem widerspenstigen Planeten die wesentliche Aufgabe des Weltalls erfülle, nämlich Götter hervorzubringen. Der Kosmos ist, nach dem französischen Wortlaut „une Machine á faire des dieux“, eine Götter hervorbringende Maschine. In dieser Hervorbringung hat die Entwicklung des göttlichen élan vital, der göttlichen Kreativität, in der Technik ihr Ziel erreicht: die göttliche Kreativität, die in den Menschen schöpferische Götter erschafft. Die mystische Identitätserfahrung war, in Bergsons Deutung, auch schon die Erfahrung der Einheit zwischen dem Schöpfer und seinem schöpferischen Geschöpf. Zur Mystik tritt hier Nietzsches Idee vom Übermenschen, von dem Bergson beeinflusst war. Mit der mystischen Bewältigung des technischen Zeitalters macht der Mensch selbst einen Übergang des Weltalls möglich, wobei diese Selbstübersteigung seine ureigene Aufgabe ist.

Die Mystik hat bei Bergson in einem durch die moderne Technik bestimmten menschlichen Leben einen überraschenden Platz erhalten. Inwieweit seine Evolutionsphilosophie mitsamt ihrer spekulativen Dimension haltbar ist, ist schwer zu sagen. In der Beziehung der Evolutionstheorie zur Technik stellt sich die Frage, ob sich der Mensch durch die Entwicklung der Technik selbst als Naturwesen übersteigt und auf diese Weise als freies Wesen zu sich selber kommt oder ob der Mensch durch die Technik auch sich selbst als Mensch übersteigt. Im ersten Fall ginge es um eine Selbstverwirklichung, im zweiten Fall um eine Art Selbstschöpfung bzw. Neuschöpfung des Menschen. In diesem Fall wird der Mensch als ein Wesen verstanden, das sich wesentlich selbst übersteigt, ein Sachverhalt, der ein wenig dem Problem der Theosis (Gott[gleich]werdung) in der christlichen Theologie ähnelt. Die Frage ist, ob diese Theosis bedeutet, dass der Mensch vergöttlicht oder in seinem vertieften Menschsein gottähnlicher wird.

Wie immer diese Fragen beantwortet werden, Bergson hat Recht, dass die Selbstrealisierung des Menschen eine Art Selbstschöpfung bzw. Selbstgestaltung in neuen, historischen Gestalten impliziert. Weiters ist ihm zuzustimmen, dass der Mensch durch die Technik neue Dimensionen in der Natur und in sich selbst realisiert und so sich selbst mit seinen Werken der Natur hinzufügt, die nicht mehr ohne Technik gedacht werden kann. In diesem Sinne kann die technische Natur als ein neues Stadium der Evolution der Natur verstanden werden. Bergson antizipiert aktuelle Thesen über das „Anthropozän“, das Zeitalter der Evolution der Natur, in der die Natur durch die Technik definitiv mitgestaltet ist. Eine ökologische Kritik dieses Denkens ist bei Bergson jedoch noch nicht ausgebildet. Sein Denken über Technik wird noch gänzlich von der 
Idee der sozialen Entwicklung bestimmt, von der Technik als Form der Caritas. Dazu gehört auch die Mystik, genauer die Mystik für normale Menschen! Die Idee, dass der neue Leib der Technik auch ökologisch beseelt werden soll, widerspricht jedoch Bergsons Konzeption keineswegs. Die Perspektive der Einheit der dynamischen Religion, die mit der Gottesidee und der Idee der Menschheit zusammenfällt, muss, wie Jaspers später vorschlagen wird, in einem Dialog der Religionen konkretisiert werden. Es ist zwar schwierig, die Idee der Weltreligionen, darunter auch die Idee des Christentums, gänzlich von der Mystik her zu bestimmen, wie Bergson dies tut; dennoch müsste die Mystik als universelle, Grenzen sprengende Erfahrung in diesem Dialog einen eigenen, spezifischen Platz erhalten. In diesem Dialog der Spiritualität eröffnet sich dann, wie von Bergson ersehnt, die Tiefendimension unseres Bewusstseins, die notwendig ist, nicht nur für die Bewältigung, sondern auch für die Beseelung der universal gewordenen technischen Kultur. Bergsons Idee der Mystik als neuer Seele unseres modernen technischen Leibes bleibt eine faszinierende Idee und stellt sowohl in theoretischer als auch in praktischer Hinsicht eine große Herausforderung dar.

\section{Biography}

Dr. Gerrit Steunebrink (1948) was a lecturer of Philosophy of Religion and Culture at the Radboud University in Nijmegen. He wrote about aesthetics, religion and metaphysics in I. Kant, G.W.F. Hegel, W. Dilthey, K. Jaspers and Th. W. Adorno. For his philosophy of culture he focused on the relations between Islam, Christianity, human rights, modernity, Turkey and Europe. After his retirement in 2013 he became an associate researcher of the Titus Brandsma Institute of the Radboud University.

\section{Bibliography}

Antes, Peter: „Mystische und prophetische Religionen: Eine gültige Unterscheidung?“, in: Religionen unterwegs. Zeitschrift der Kontaktstelle für Weltreligionen in Österreich 4 (1/1998), pp. 4-8.

Barnard, William, G.: "Turning into other worlds: Henri Bergson and the Radio Reception Theory of Consciousness", in: Alexandre Lefebvre/Melanie White (ed.): Bergson, Politics, and Religion. Durham/London: Duke University Press 2012, pp. 281-298.

Bergson, Henri: Les deux sources de la morale et de la religion, in: ders.: Oeuvres. Paris: Presses Universitaires de France 1970, pp. 979-1247 (German: Die beiden Quellen der Moral und der Religion, translated by Eugen Lerch. Jena: Diederich 1933). 
Germino, Dante: "Preliminary Reflections on the Open Society: Bergson, Popper, Voegelin", in: Dante Germino/Klaus von Beyme (ed.): The Open Society in Theory and Praxis. The Hague: Martinus Nijhoff 1974, pp. 1-25.

Gontier, Thierry: "The Open Society, from Bergson to Voegelin", in: APSA 2012 Annual Meeting Paper. 2012. Online verfügbar: SSRN, URL: http://ssrn.com/abstract=2111016 (date of last access: 09.08.2019).

Jaspers, Karl: Vom Ursprung und Ziel der Geschichte. München: Piper ${ }^{8} 1983$ [1949].

Kolakowski, Leszek: Bergson. Oxford/New York: Oxford University Press 1985.

Lawlor, Leonad/Moulard, Valentine: "Henri Bergson”, in: The Stanford Encyclopedia of Philosophy (Summer 2016 Edition), Edward N. Zalta (ed.), URL: https://plato. stanford.edu/archives/sum2016/entries/bergson/ (date of last access: 09.08.2019).

Lefebvre, Alexandre/White, Melanie (ed.): Bergson, Politics and Religion. Durham/ London: Duke University Press 2012.

Lévi-Strauss, Claude: Le totémisme aujourd'hui, in: Oeuvres. Paris: Éditions Gallimard 2008, pp. 447-551.

Maréchal, Joseph: "Ontology and Theology of Christian Mysticism”, in: Richard Woods (ed.): Understanding Mysticism. New York: Image Books 1980, pp. 469-476. 